OPEN ACCESS

Edited by:

Benjamin Gourion, Laboratoire Interactions Plantes-

Microorganismes, France

Reviewed by:

Maria Jose Soto,

Experimental Station of Zaidín (EEZ)

Spain

Viktor E. Tsyganov,

All-Russian Research Institute of

Agricultural Microbiology of the

Russian Academy of Agricultural

Sciences, Russia

*Correspondence:

Pierre Frendo

Pierre.FRENDO@univ-cotedazur.fr

${ }^{t}$ These authors share first authorship

Specialty section:

This article was submitted to Plant Microbe Interactions,

a section of the journal

Frontiers in Plant Science

Received: 17 September 2019 Accepted: 29 January 2020

Published: 03 March 2020

Citation:

Yang L, El Msehli S, Benyamina S, Lambert A, Hopkins J, Cazareth J, Pierre O, Hérouart D, Achi-Smiti $S$, Boncompagni $E$ and Frendo $P$ (2020) Glutathione Deficiency in Sinorhizobium meliloti Does Not Impair Bacteroid Differentiation But Induces Early Senescence in the Interaction With Medicago truncatula.

Front. Plant Sci. 11:137. doi: $10.3389 /$ fpls.2020.00137

\section{Glutathione Deficiency in} Sinorhizobium meliloti Does Not Impair Bacteroid Differentiation But Induces Early Senescence in the Interaction With Medicago truncatula

\author{
Li Yang ${ }^{1 \dagger}$, Sarra El Msehli ${ }^{2 \dagger}$, Sofiane Benyamina ${ }^{1}$, Annie Lambert ${ }^{1}$, Julie Hopkins ${ }^{1}$, \\ Julie Cazareth ${ }^{3}$, Olivier Pierre ${ }^{1}$, Didier Hérouart ${ }^{1}$, Samira Achi-Smiti ${ }^{2}$, Eric Boncompagni ${ }^{1}$ \\ and Pierre Frendo ${ }^{1 *}$ \\ 1 Université Côte d'Azur, INRA, CNRS, ISA, Sophia-Antipolis, France, ${ }^{2}$ Laboratoire de Physiologie Végétale, Faculté des \\ Sciences de Tunis, Campus Universitaire El Manar II, Tunis, Tunisia, ${ }^{3}$ Institut de Pharmacologie Moléculaire et Cellulaire, \\ CNRS UMR 7275, Université Côte d'Azur, Valbonne, France
}

Under nitrogen-limiting conditions, legumes are able to interact symbiotically with bacteria of the Rhizobiaceae family. This interaction gives rise to a new organ, named a root nodule. Root nodules are characterized by an increased glutathione (GSH) and homoglutathione (hGSH) content compared to roots. These low molecular thiols are very important in the biological nitrogen fixation. In order to characterize the modification of nodule activity induced by the microsymbiont glutathione deficiency, physiological, biochemical, and gene expression modifications were analyzed in nodules after the inoculation of Medicago truncatula with the SmgshB mutant of Sinorhizobium meliloti which is deficient in GSH production. The decline in nitrogen fixation efficiency was correlated to the reduction in plant shoot biomass. Flow cytometry analysis showed that SmgshB bacteroids present a higher DNA content than free living bacteria. Live/dead microscopic analysis showed an early bacteroid degradation in SmgshB nodules compared to control nodules which is correlated to a lower bacteroid content at 20 dpi. Finally, the expression of two marker genes involved in nitrogen fixation metabolism, Leghemoglobin and Nodule Cysteine Rich Peptide 001, decreased significantly in mutant nodules at $20 \mathrm{dpi}$. In contrast, the expression of two marker genes involved in the nodule senescence, Cysteine Protease 6 and Purple Acid Protease, increased significantly in mutant nodules at $10 \mathrm{dpi}$ strengthening the idea that an early senescence process occurs in SmgshB nodules. In conclusion, our results showed that bacterial GSH deficiency does not impair bacterial differentiation but induces an early nodule senescence.

Keywords: Medicago truncatula, Sinorhizobium meliloti SmgshB, glutathione, bacteroid differentiation, senescence 


\section{INTRODUCTION}

Nitrogen $(\mathrm{N})$ is the major macronutrient needed for plant growth. However, whereas $\mathrm{N}$ is the most abundant element in the atmosphere, it is generally the most limiting plant nutrient in soils. Legume plants are able to interact symbiotically with rhizobia to perform nitrogen fixing symbiosis (NFS). In these mutualistic associations, bacteria deliver nitrogen source to the plants while plants provide bacteria with photosynthates, nutrients required for rhizobium metabolism and a specialized ecological niche that favors their growth. Indeed, the nitrogenfixing symbiosis involves the de novo formation of a new organ, the nodule, associated to a reprogramming of the plant development and metabolism. This allows the intracellular accommodation of several thousands of differentiated bacteria, within nodule cells, called bacteroids which reduce atmospheric nitrogen. Amongst the multiple regulatory processes involved in the setup and the regulation of NFS, a modification of the cellular redox state of the plant partner is observed during the plant infection and nodule functioning (Puppo et al., 2013). Reactive oxygen species production is detected in root hairs in response to nodulation factors (Cárdenas et al., 2008), the root infection process (Santos et al., 2001; Jamet et al., 2007) and in the nodule differentiation zone in Medicago truncatula nodules (Andrio et al., 2013). The implication of the ROS in the symbiotic interaction between legumes and rhizobia has been demonstrated using genetic approaches (Marino et al., 2011; Arthikala et al., 2014). Genetic alteration of the expression of respiratory burst oxidative homologs (Rbohs), proteins involved in the ROS production, significantly modifies the symbiosis between legume plants and the rhizobia (Marino et al., 2011; Marino et al., 2012). Similarly, nitric oxide (NO) production is also detected in the NFS (Hichri et al., 2015). It is required for the optimal establishment of the symbiotic interaction (Hichri et al., 2015; Berger et al., 2019). In mature nodules, NO has a beneficial metabolic function for the maintenance of the energy status under hypoxic conditions, but has also been shown to inhibit nitrogen fixation and to trigger nodule senescence. NO has also been shown to regulate the glutathione (GSH) synthesis in roots (Innocenti et al., 2007).

The root nodules contain significantly higher contents of low molecular thiols, GSH and homoglutathione (hGSH), in comparison to roots (Frendo et al., 2013a; Frendo et al., 2013b). GSH is the most abundant low-molecular weight thiol in cells and has several well-known functions. For example, it acts as a redox buffer and as a signaling molecule, and it is also involved in the detoxification of radicals and xenobiotics (Rouhier et al., 2008; Foyer and Noctor, 2011). GSH is synthesized by a two-step process. In the first step, glutamate and cysteine are conjugated by $\gamma$-glutamyl cysteine synthetase $(\gamma$-ECS) to form $\gamma$-glutamyl cysteine $(\gamma$-EC). In a second step, glycine is added to $\gamma$-EC to form GSH in a reaction catalyzed by glutathione synthetase (GSHS). Alternatively, homoglutathione synthetase (hGSHS) catalyzes the addition of $\beta$ alanine to $\gamma$-EC to form hGSH. Genetic and pharmacological approaches showed that the GSH pool produced by the plant partner is important to allow the nodule development and an efficient biological nitrogen fixation (BNF) (Frendo et al., 2005;
El Msehli et al., 2011). On the bacterial side, the use of mutants deficient in the GSHS-encoding gene (gshB) impairs the BNF efficiency during the symbiosis between Medicago sativa/S. meliloti strain 1021, Phaseolus vulgaris/Rhizobium tropici, P. vulgaris/Rhizobium etli, and Pisum sativum/Rhizobium leguminosarum (Harrison et al., 2005; Muglia et al., 2008; Taté et al., 2012; Cheng et al., 2017). The reduced BNF observed with the $g s h B$ mutant strain was correlated with nodule and bacteroid early senescence in M. sativa and P. vulgaris (Harrison et al., 2005; Muglia et al., 2008; Taté et al., 2012). In parallel, recycling of GSH by glutathione reductase was shown to be required to maintain an effective nitrogen fixation (Tang et al., 2018). Finally, a deficiency in bacteroid differentiation was observed for a S. meliloti (strain 2011) mutant for glutaredoxin 1 (Grxl), a glutaredoxin involved in the deglutathionylation of proteins using GSH as a cofactor, in the symbiotic interaction with $M$. truncatula (Benyamina et al., 2013). Harrison and colleagues (2005) also suggested that glutathione deficiency in S. meliloti strain 1021 interferes with bacteroid differentiation in M. sativa (Harrison et al., 2005) but this hypothesis was not carefully investigated.

In this context, we hypothesized that the genome background of S. meliloti (strain 1021 versus strain 2011) or the genome background of the plant (M. sativa versus M. truncatula) could modify the outcome of the interaction between the two partners. With this in mind, we intended to characterize the impact of the bacterial GSH deficiency on the bacteroid differentiation and the nodule functioning during the symbiotic interaction between $M$. truncatula and the strain 2011 of S. meliloti. Physiological, biochemical, cellular and genetic markers were used to describe the nodule functioning at 10 and 20 days after plant inoculation, two early time points of nodule functioning. Our results show that bacterial GSH deficiency does not affect bacteroid differentiation. However, bacterial GSH deficiency induces an early nodule senescence process in $M$. truncatula already detectable at $10-\mathrm{d}$ post-infection.

\section{MATERIALS AND METHODS}

\section{Plant Material, Bacterial Strains and Growth Conditions}

A mutation in $g s h B$ (Smc00419) of S. meliloti 2011 was made by plasmid integration using single-crossover. A 680-bp gshB fragment was PCR-amplified using primers SMc00419-F (5'AAGAATTCCCATGTTTCGGGCATCACC-3') and SMc00419-R (5'-AACGATC GCTGCAGTGCATGTTGGAGC GAGAATC-3'). The fragment was cloned into EcoRI-PvuI sites of pSUP202, resulting in plasmid pSUP202gshB. Plasmid pSup202gshB was transferred from E. coli to S. meliloti 2011 and recombined into the genomic gshB region via single crossover to give the mutant strain SmgshB in S. meliloti 2011 genetic background. The mutants were screen for resistance to tetracyclin $(10 \mu \mathrm{g}$ per $\mathrm{ml})$ and streptomycin $(100 \mu \mathrm{g}$ per $\mathrm{mL})$. The SmgshB mutant strain in Sm 2011 genetic background was validated by HPLC for the lack of GSH as described previously (Harrison et al., 2005). The delayed growth phenotype observed for the mutant strain was complemented by the addition of GSH 
(Figure S1). M. truncatula ecotype Jemalong A17, S. meliloti 2011 (wild type), and S. meliloti 2011 gshB (SmgshB) strains were grown as described (Harrison et al., 2005). M. truncatula seeds were then spread on $0.4 \%$ agar gel at $4^{\circ} \mathrm{C}$ for 2 days for vernalization and germinated at $16^{\circ} \mathrm{C}$ for 1 day. Seedlings were afterwards growing in vitro on Fahraeus medium (BoissonDernier et al., 2001). Each seedling was inoculated with $200 \mu \mathrm{l}$ of S. meliloti at an O.D.600nm of 0.05. All the plants used in this study were grown in a 16 -h light $\left(23^{\circ} \mathrm{C}\right)$ : 8 -h dark $\left(20^{\circ} \mathrm{C}\right)$ photoperiod. Nodules were harvested at 10 - and 20 -days postinoculation (dpi). At least three independent biological repetitions were analyzed.

\section{Nitrogenase Activity Assay}

Nitrogen fixation activity was determined by $\mathrm{C}_{2} \mathrm{H}_{2}$ reduction assay (ARA), using a gas chromatograph. Nodulated roots at 10 and 20 dpi were detached from plants and put inside sealed vials. In each vial, $10 \%$ of the atmosphere was substituted with acetylene. After $1 \mathrm{~h}$ of incubation at $28^{\circ} \mathrm{C}$, gas samples were analyzed with a gas chromatograph system (Agilent GC $6890 \mathrm{~N}$; Agilent Technologies, Massy, France). Twelve plants were tested per replicate and at least three different biological replicates were analyzed.

\section{Flow Cytometry}

S. meliloti bacteroids were purified as previously described (Trinchant et al., 2004) using a $320 \mathrm{mOsM}$ extraction buffer. Bacterial cells were fixed by heat treatment $\left(70^{\circ} \mathrm{C}, 15 \mathrm{~min}\right)$ and stained with $10 \mu \mathrm{g} / \mathrm{mL}$ propidium iodide (PI). They were then subjected to flow cytometry analysis with a LSRII Fortessa flow cytometer (BD BioSciences, Rungis, France). Bacterial DNA content was determined from PI fluorescence at 585-625 nm after excitation at $565 \mathrm{~nm}$ with a single laser line. Single events were recorded and analyzed with BD FACSDiva v6.1.3 (BD BioSciences) and Kaluza v1.2 software (Beckman Coulter, Villepinte, France), respectively. Experiments were performed with biological triplicates and the recording of more than 200,000 events for each sample to ensure the statistical robustness of each assay (Ribeiro et al., 2017).

\section{Live/Dead Staining and Microscopic Observations}

Nodulated roots and nodules at 10 and 20 dpi, respectively, were harvested and embedded in $4 \%$ agarose. Nodule sections of $70 \mu \mathrm{m}$ were prepared with a Vibratome HM650V (Thermo Fisher Scientific, Illkirch-Graffenstaden, France). Afterwards, nodule sections were stained with SYTO $^{\mathrm{TM}} 9$ and propidium iodide (PI) (LIVE/DEAD BacLight Bacterial Viability Kit, Thermo Fisher scientific) for $20 \mathrm{~min}$ as previously described (Haag et al., 2011). Sections were then mounted on slides and visualized with a laser scanning confocal microscope LSM880 (Carl Zeiss, Marly le Roi, France). The SYTO ${ }^{\text {TM }} 9$ and PI were sequentially excited by $488 \mathrm{~nm}$ Argon and $561 \mathrm{~nm}$ HeNe laser lines, separately. Both fluorescence signals were recorded on separated detectors. All images were treated with Fiji software (Schindelin et al., 2012). Samples are from at least 30 nodules of 15 plants from three independent replicates.
Quantitative Reverse Transcriptase PCR (RT-qPCR)

Young nodules (10 dpi) and mature nodules (20 dpi) were harvested. Total RNA was extracted from $100 \mathrm{mg}$ of nodules with Trizol (Invitrogen, Illkirch-Graffenstaden, France) and cDNA was synthesized with the Omniscript RT kit (Qiagen, Les Ulis, France) after a DNAse treatment of total RNA. Absence of genomic contamination was ensured by control PCR with intron-spanning primers. Quantitative PCR was performed using AriaMx Real-time PCR system (Agilent, Les Ulis, France). All data were normalized with two constitutively expressed genes, Mtc27 and A38 ( $M$. truncatula) for the $M$. truncatula genes, and $16 S$ ribosomal RNA and Polyribonucleotide nucleotidyltransferase for the $S$. meliloti genes. The primer sequences used for PCR are described in Table S1. Each RTqPCR reaction for each of the three biological experiments was performed in triplicate. The expression fold change was calculated as $2^{-\Delta \Delta C t}$ method (Livak and Schmittgen, 2001).

\section{Statistical Analyses}

Our data were reported as mean \pm standard error. The significance of the results was assessed using the MannWhitney nonparametric test, which allows the comparison of small quantitative samples. When the number of samples was enough to define a normal distribution (30 samples), the results were assessed using Student's t-test.

\section{RESULTS}

\section{Analysis of Plant Phenotype and Biological Nitrogen Fixation}

To test the phenotype of the S. meliloti SmgshB strain, we have analyzed nodule functioning at 10 - and 20 - dpi which corresponds to young and mature nodules in vitro growth conditions. The phenotypes of the plants and the root nodules were examined, and the nitrogen fixation activity was analyzed by ARA (Figure 1). The nitrogen fixation efficiency was significantly reduced by $53 \%$ and $21 \%$ in SmgshB inoculated plants compared to control plants at 10 and 20 dpi, respectively (Figure 1A). A significant lower number of nodules was observed for SmgshB inoculated plants compared to the control plants at $10 \mathrm{dpi}$ (Figure S2) and nodule size was not different at 10 or 20 dpi (Figure S2). Phenotypic analysis of root nodules showed that SmgshB nodules presented a less pronounced pink color compared to control ones and present an extended green color on the distal part of nodule at 20 dpi (Figure 1B). A significant diminution of shoot dry weight biomass and a reduced plant development were observed in SmgshB inoculated plants compared to the control plants at $20 \mathrm{dpi}$ (Figures 1C and S3). In conclusion, SmgshB inoculated plants present an altered growth phenotype compared to the control plants.

\section{Analysis of Bacteroid Differentiation Using Flow Cytometry}

The bacteroid differentiation is correlated to an increase in size and in DNA content of the bacteroid compared to the bacterium 

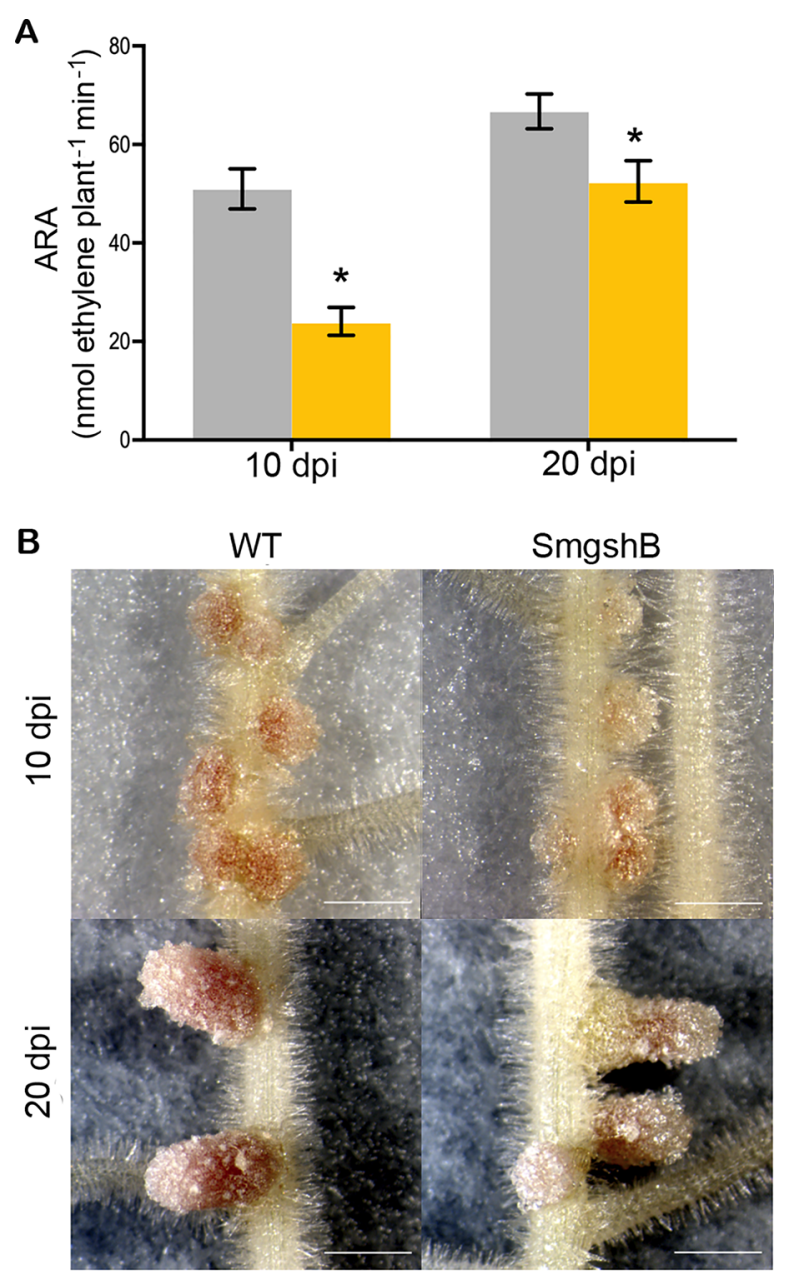

C

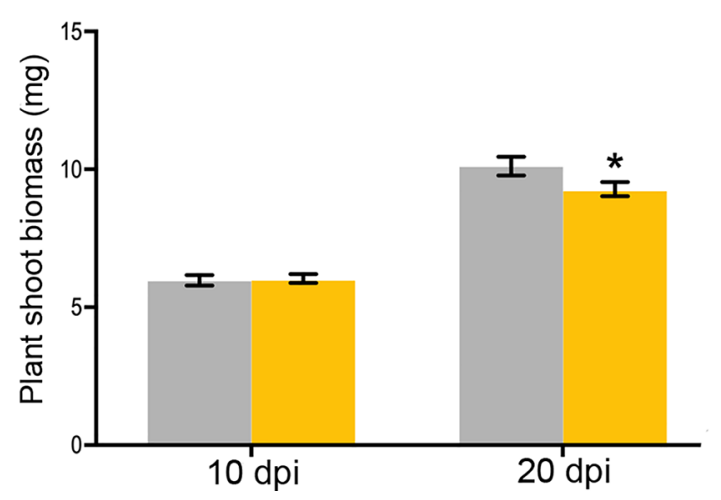

FIGURE 1 | Physiological analysis of the symbiosis between Medicago truncatula and Sinorhizobium meliloti SmgshB mutant strain. (A) Nitrogen fixation capacity of plants with WT strain (grey) and the mutant SmgshB (yellow) at 10- or 20-d post-inoculation (dpi), measured by the acetylene reduction assay per plant (nanomoles of ethylene produced per minute of incubation and per plant). (B) Phenotype of nodules formed with indicated strains at 10- or 20- dpi. Scale bars $=1 \mathrm{~mm}$. (C) Dry weight shoot mass of M. truncatula inoculated with WT (grey) and SmgshB mutant (yellow) strains at 10 or 20 dpi. Error bars are standard errors and * indicates a statistically significant difference $(P<0.05 ; n=15)$.
(Mergaert et al., 2006). These criteria could be used to differentiate bacteria from bacteroids using flow cytometry (Ribeiro et al., 2017). To test whether GSH deficiency in the bacteria impairs the bacteria differentiation, we have analyzed the bacteroid and bacterial content in nodules from control plants and SmgshB inoculated plants at 10 and 20 dpi using flow cytometry (Figure 2). Bacterial cells showing DNA endoreduplication were observed in both control and SmgshB nodules at 10 and $20 \mathrm{dpi}$ (Figure 2A). The nitrogen-fixing bacteroid (DNA content 4C and more) proportion in control and SmgshB nodules was similar at around $40 \%$ at 10 dpi (Figure 2B). In contrast, the proportion of bacteroids was significantly lower in SmgshB nodules than in control ones at $20 \mathrm{dpi}$, with a reduction of $40 \%$ in bacteroid content compared to the control (Figure 2B). Therefore, these results show that bacteroid differentiation occurs in SmgshB nodules in a similar way that in control nodule at $10 \mathrm{dpi}$. However, the lower content of bacteroids in SmgshB nodules compared to control ones shows that the nodule bacteroid content decreases in mature nodules. This decrease of bacteroid content in SmgshB nodules may be associated to a reduced bacterial differentiation during nodule aging or to a faster bacteroid degradation compared to control nodules.

\section{Analysis of Bacteroid Survival Using Live/ Dead Staining in Nodule}

To analyze the survival of bacteroids in the SmgshB nodules, the live/dead staining was used at 10 and 20 dpi (Figure 3). Permeable bacteroid cell membranes that are considered dead or dying and plant nucleus are stained red with propidium iodide (PI), while bacteroids with intact membranes are green due to SYTO $^{\text {TM }} 9$ staining of nucleic acids (Haag et al., 2011). No significant difference was observed at $10 \mathrm{dpi}$ between the SmgshB and control bacteroids (Figures 3A, C). At $20 \mathrm{dpi}$, the nodules are more elongated with a larger zone III in control nodules. No significant bacteroid degradation was observed in the control nodules (Figure 3F). In contrast, SmgshB nodules presented a strong alteration of the nodule cellular structure with numerous dead bacteroids and empty plant cells in zone III (Figure 3H). These results show that the cellular structure of SmgshB nodule is not modified at early time point but present significant degradation at $20 \mathrm{dpi}$. This suggests that senescence occurs ealier in SmgshB nodules than in control nodules.

\section{Expression of Nodule Marker Genes in SmgshB Nodules}

In order to analyze whether the cellular and biochemical alterations observed in the SmgshB nodules could be correlated to modifications in the nodule genetic program, the expression level of plant gene markers was analyzed by RT-qPCR. These genes are involved either in the functioning of nodules or are genes potentially markers of senescence. The expression of eight marker genes was measured to analyze the SmgshB nodule transcriptomic modifications (Figure 4). The expression of marker genes expressed in the nodule differentiation zone (Thioredoxin s1, Trx s1), in the nitrogen fixation zone (Nodule Cysteine Rich Peptide, NCRO01 and leghemoglobin, Lb) and during nodule senescence (Cysteine Proteases 6, CP6, Vacuolar Processing Enzyme, VPE, and Purple Acid Protease, PAP) was 


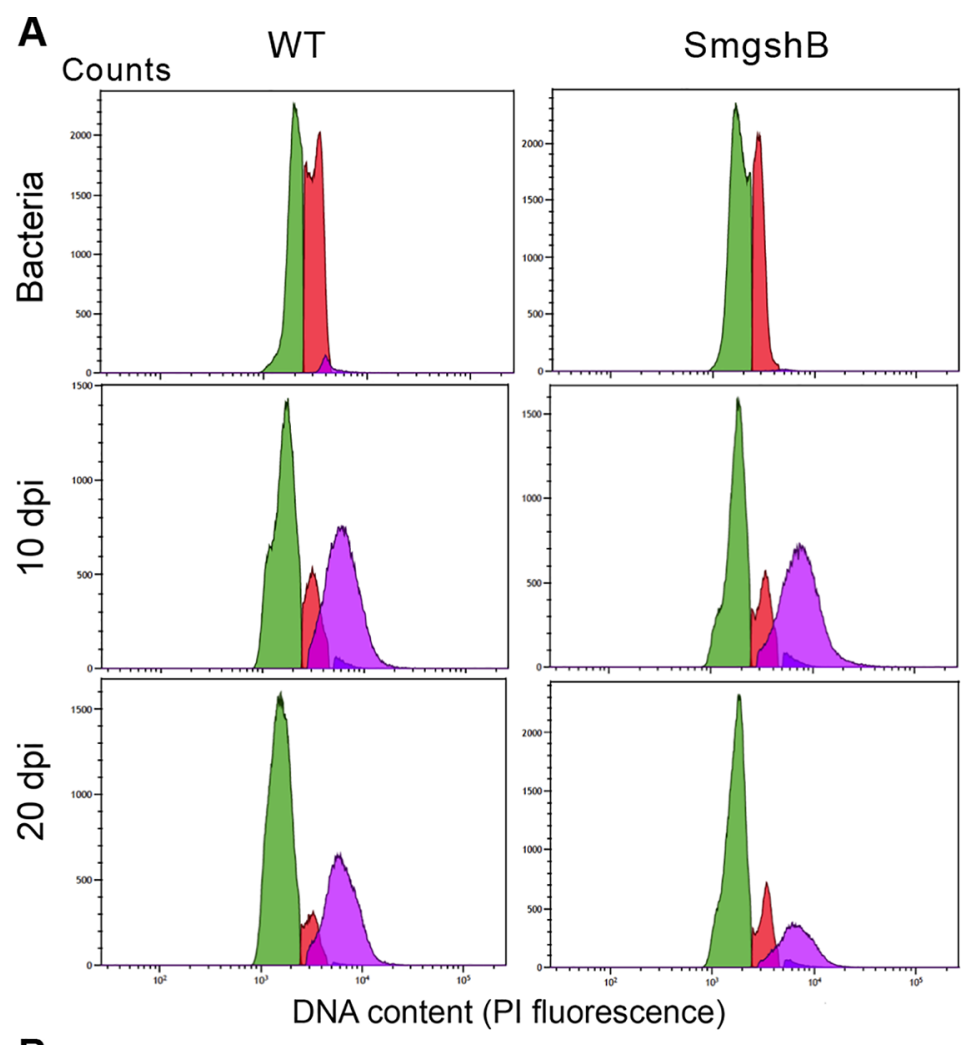

B

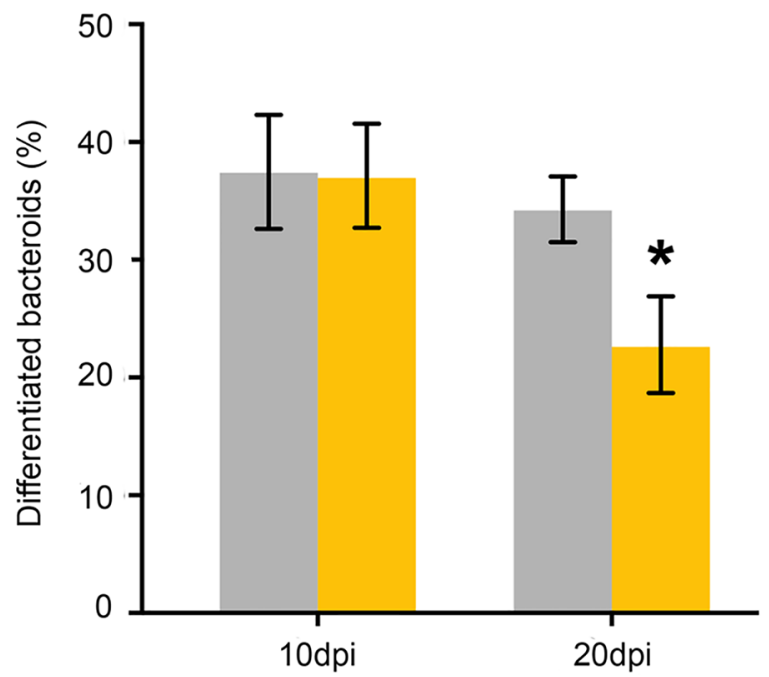

FIGURE 2 | Flow cytometry analysis of SmgshB bacteroids. (A) DNA content (PI florescence) of bacteria and bacteroids extracted from nodules obtained with S. meliloti 2011 strain or SmgshB mutant strain after 10 or 20 dpi. DNA content of bacterial and bacteroid populations is indicated in color as: green: 1C, red: 2C, purple: $4 \mathrm{C}$ and more. The datasets shown are representative of trends observed in three independent experiments. (B) Percentage of bacteroids with a large SSC (side scatter) value and DNA value of $4 \mathrm{C}$ and more. The values shown are the means \pm SE of three independent replicates (at least two plants per replicate). Significance was determined using the Mann-Whitney nonparametric test $(P<0.05 ; n=3)$. The * indicates a statistically significant difference $(P<0.05 ; n=3)$.

studied in control and SmgshB nodules. Moreover, the expression of two bacterial genes nifD (nitrogenase molybdenum-iron protein alpha chain) and nifH (nitrogenase iron protein), encoding nitrogenase subunits and expressed in nitrogen-fixing bacteroids, was analyzed in control and SmgshB nodules. $\operatorname{Tr} x$ s1 is specifically expressed during the symbiotic interaction between M. truncatula and S. meliloti (Alkhalfioui et al., 2008) and its expression is localized in the nodule infection zone (Ribeiro et al., 2017). NCR001 is considered as a specific marker of the nitrogen fixation zone (Mergaert et al., 2003) like 


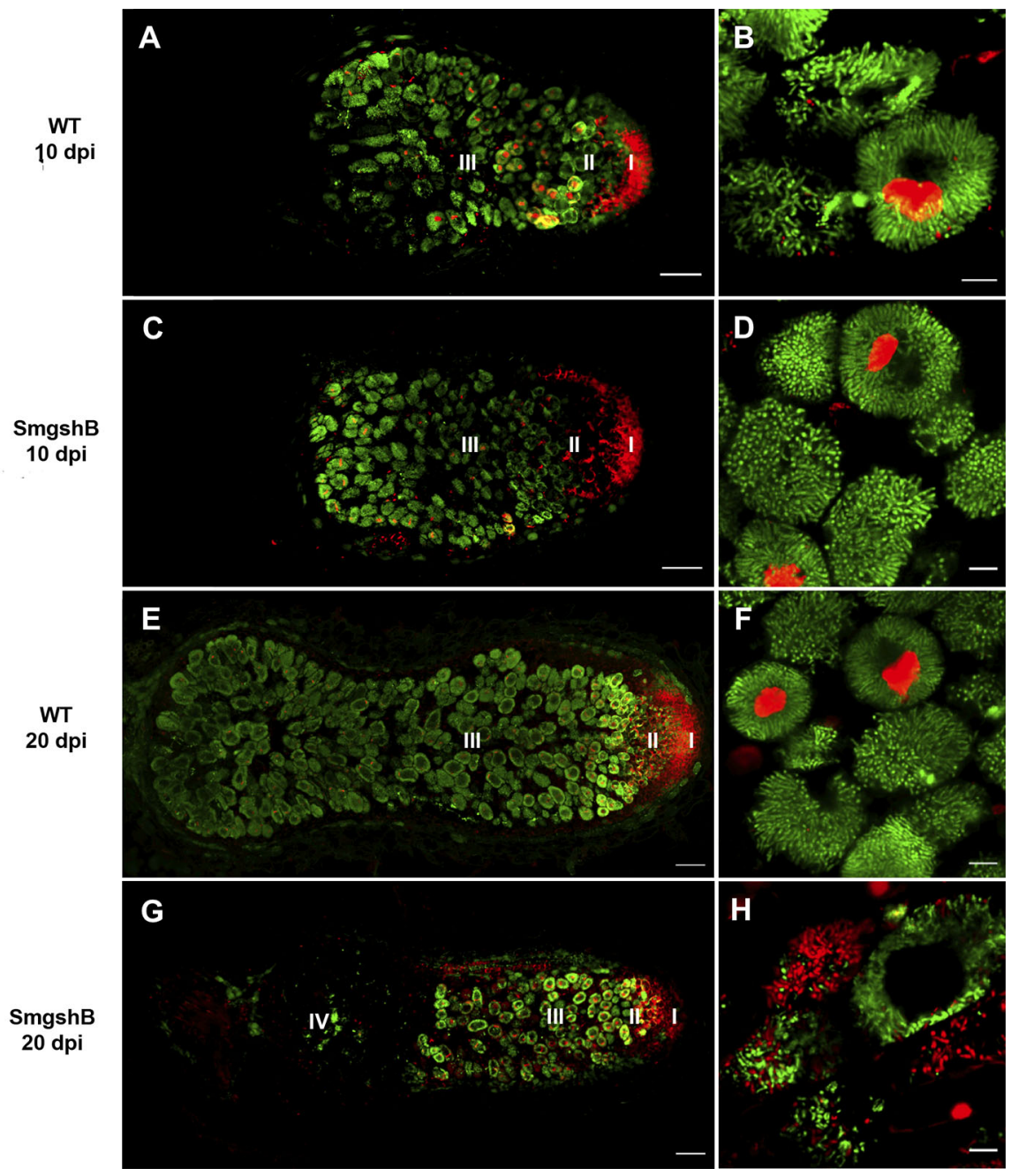

FIGURE 3 | Live/dead microscopic analysis of SmgshB nodules. M. truncatula plantlets were inoculated with either WT or SmgshB strains. Bacteroid viability of nodule sections determined live/dead staining by SYTO9 and PI dyes at 10 and 20 dpi. Viable bacteria/bacteroid are stained green by SYTO9 and damaged bacteroids are stained red by PI. The different zones of the nodule are indicated: I, the meristem; II, the infection zone; III, the nitrogen fixation zone; and IV, the senescent zone. Panel (B, D, F, H) represent infected cells in zone III. The images shown are representatives of at least 30 nodules of 15 plants from three independent replicates. Scale bars $=100 \mu \mathrm{m}(\mathbf{A}, \mathbf{C}, \mathbf{E}, \mathbf{G})$ or $=10 \mu \mathrm{m}(\mathbf{B}, \mathbf{D}, \mathbf{F}, \mathbf{H})$.

Leghemoglobin (Mergaert et al., 2003; Ott et al., 2005). The expression of CP6 and VPE, two genes encoding cysteine proteases, is induced during developmental- and stress-induced nodule senescence (Pierre et al., 2014). Finally, the expression of $P A P$ is induced during the nodule senescence process in $M$. truncatula (Xi et al., 2013). In silico analysis of this PAP isoform showed that it is significantly more expressed in the nodule than in roots and significantly more expressed in the nitrogen fixation zone than in the nodule meristematic and differentiation zones (Table S2).

Analysis of $\operatorname{Tr} x s 1$ expression showed that $\operatorname{Tr} x \mathrm{~s} 1$ expression was not significantly modified in SmgshB nodules compared to control nodules at 10 and 20 dpi suggesting a normal functioning of the nodule infection zone. The expression levels of NCR001 and $L b$ are significantly down-regulated in SmgshB nodules in comparison to control ones at $20 \mathrm{dpi}$. The expression of nifH and nifD was similar in SmgshB and control nodules at $10 \mathrm{dpi}$ and nifH was significantly down-regulated in SmgshB nodules in comparison to control ones at $20 \mathrm{dpi}$. Regarding the senescence related gene markers, CP6, VPE, and PAP expression are, conversely, significantly up-regulated in SmgshB nodules compared to control nodules. The similar expression of nifH and nifD at 10 dpi strengthens the flow cytometry analysis and shows that the SmgshB bacteria are able to differentiate into bacteroids. Moreover, the down-regulation of the genes involved in BNF efficiency is correlated to the induction of senescence related genes, confirming that an early senescence occurs in SmgshB nodules. 

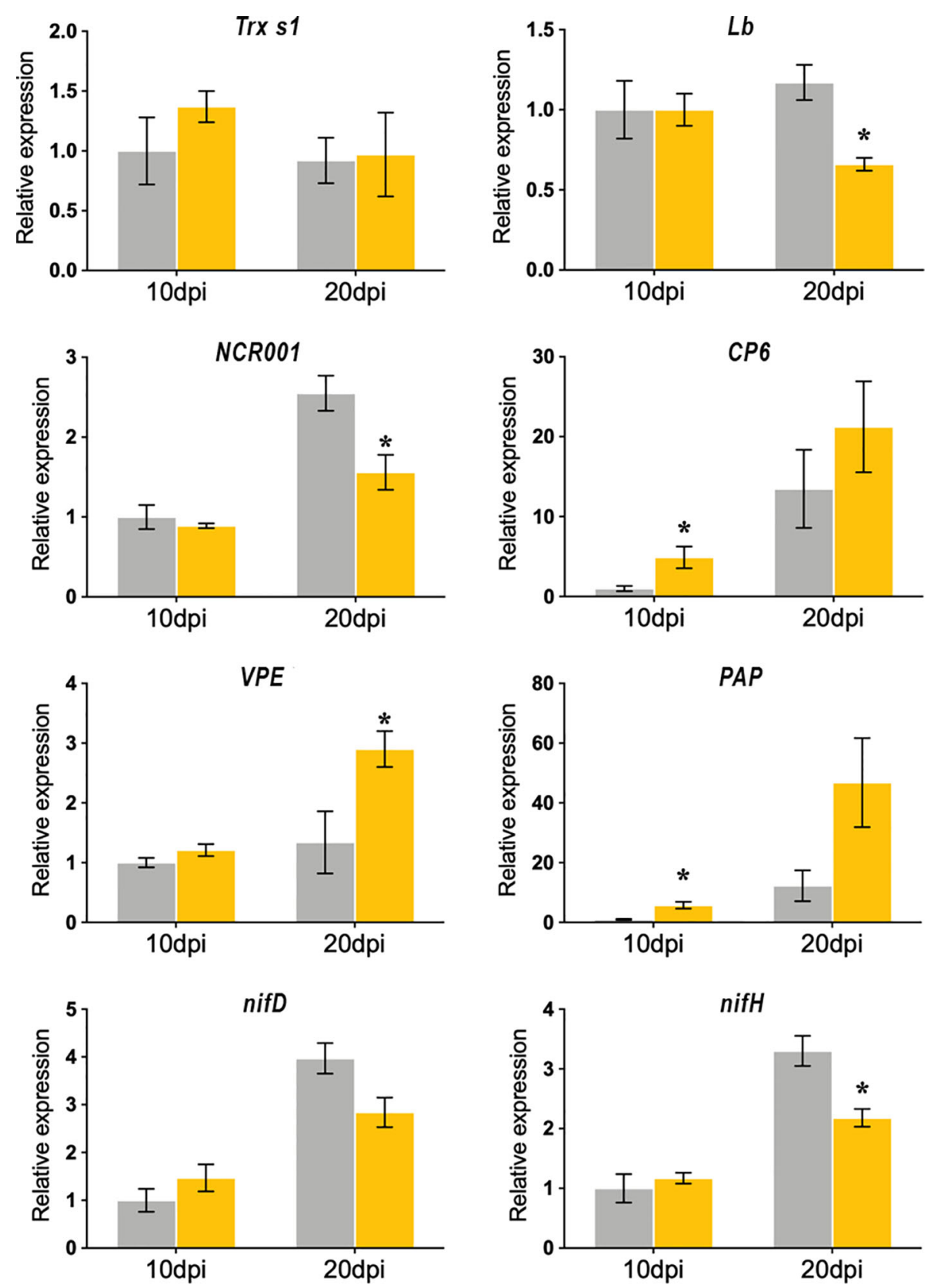

FIGURE 4 | Quantitative RT-PCR analysis of nodule gene expression markers in M. truncatula nodules inoculated with WT (grey) and SmgshB strains (yellow). The expression levels of Nodule Cysteine Rich 001 (NCR001), Leghemoglobin (Lb), Thioredoxin s1 (Trx s1), Cysteine Protease 6 (CP6), Vacuolar Processing Enzyme (VPE), Purple Acid Phosphatase (PAP), nifD (nitrogenase molybdenum-iron protein alpha chain) and nifH (nitrogenase iron protein). were analyzed in nodules. Data (technical triplicates of three biological samples) are reported as mean \pm standard error. * indicates a statistically significant difference relative to the control $(P<0.05$; $\mathrm{n}=3)$

\section{DISCUSSION}

The symbiotic interaction established between legumes and bacteria allows the plant to satisfy its needs in nitrogen. The use of a bacterial strain deficient in GSH production alters significantly the biological nitrogen fixation and the growth of M. sativa, P. vulgaris, and P. sativum during NFS (Harrison et al.,
2005; Muglia et al., 2008; Taté et al., 2012; Cheng et al., 2017). Moreover, the electronic microscopy analyses suggested that SmgshB mutant strain is partially impaired in the bacteroid differentiation (Harrison et al., 2005). More recently, the glutaredoxin SmGrx1 mutant in S. meliloti strain 2011 showed clearly a deficiency in bacteroid differentiation. Glutaredoxins (Grxs) are small ubiquitous redox enzymes that are involved in 


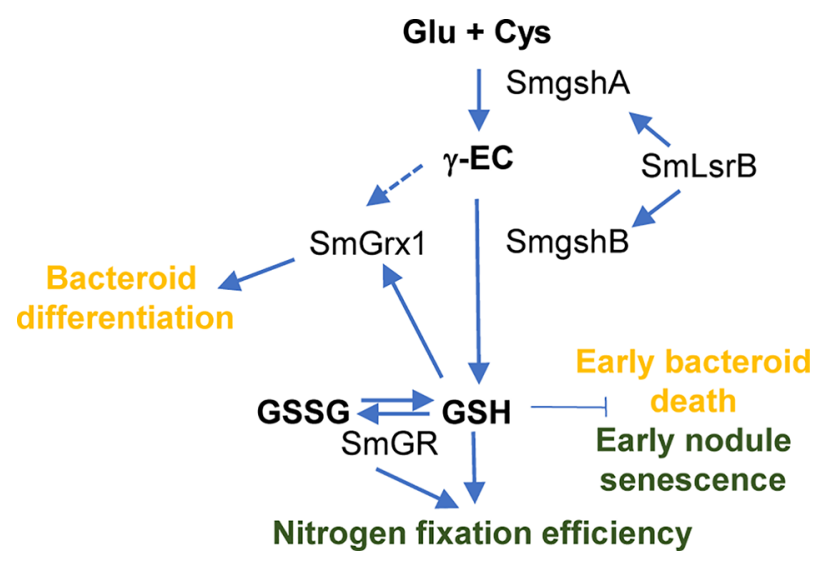

FIGURE 5 | General overview of GSH role in S. meliloti bacteroid. GSH plays a central role in the redox networks of glutaredoxin (SmGrx1) and SmLsrB. Defect in GSH accumulation lead to an early nodule senescence whereas deletion in Grx1 impairs bacteroid differentiation. The green color indicates general effects on the root nodule and the orange color indicates direct effects on the bacteroid. Dashed arrow indicates the potential role of $\gamma$-EC on SmGrx1 in absence of GSH.

the reduction of oxidative modifications using GSH (Benyamina et al., 2013). Thus, our hypothesis is that GSH produced by the strain 2011 is necessary to allow normal bacteroid differentiation in nodules formed on $M$. truncatula. In this context, we tested the symbiotic interaction between $M$. truncatula and the GSH deficient-SmgshB mutant constructed in S. meliloti strain 2011 at 10 and $20 \mathrm{dpi}$, two early time points of nodule nitrogen fixation, using a multidisciplinary approach.

The nitrogen fixation efficiency was significantly impaired in plants inoculated with SmgshB strain (Figure 1). However, this reduction is less noticeable than in plants inoculated with SmGrx1 (Benyamina et al., 2013). Moreover, at $20 \mathrm{dpi}$, elongated nodules are observed suggesting that the nodule meristem is more active in plants inoculated with SmgshB strain than in plants inoculated with SmGrx1 strain (Benyamina et al., 2013). In M. truncatula, the bacteroids present a terminal differentiation accompanied with a DNA endoreduplication and an arrest in cell division coupled to an increase in cell size (Ribeiro et al., 2015). The flow cytometry analysis of SmgshB bacteroids shows that DNA endoreduplication occurs in SmgshB bacteroid and no significant difference was observed between SmgshB nodules and control ones at $10 \mathrm{dpi}$ (Figure 2). In contrast, the number of terminally differentiated bacteroids is significantly lower in SmgshB nodules than in control ones at 20 dpi suggesting that there is a faster degradation or a slower formation of bacteroids in SmgshB nodules than in control ones. The live/dead experiments allow us to analyze the viability of the bacteroids in the root nodules. Such live/dead labelling shows an early death of bacteroids in SmgshB nodules compared to control ones at $20 \mathrm{dpi}$ (Figure 3).

Gene expression analysis showed that at $10 \mathrm{dpi}$, genes expressed in the nitrogen fixing zone, NCR001 and $L b$, are similarly expressed in SmgshB and control nodules suggesting that the gene expression in the nitrogen fixing zone is not significantly affected (Figure 4). A similar result is observed for $\operatorname{Tr} x$ s1, a gene marker for the nodule infection zone, and VPE. Similarly, expression of nifH and nifD genes was similar in SmgshB and control nodules strengthening that SmgshB bacteroid differentiation occurs properly at $10 \mathrm{dpi}$. In contrast, significant higher expression of $C P 6$ and $P A P$ is already observed in SmgshB nodules compared to control ones at $10 \mathrm{dpi}$. This result showed that in our plant growth conditions, CP6 and PAP are the earlier senescence marker genes whose expression is significantly modified, suggesting that there is an early development of senescence in SmgshB nodules. At $20 \mathrm{dpi}$, there is a significant modification in the expression of different genes. NCR001, $L b$, and nifH are less expressed in SmgshB nodules compared to control ones showing a correlation between the diminution of the nitrogen fixation efficiency and the reduction of the expression of genes expressed in the nitrogen fixation zone. Despite a strong increase of CP6 expression in 20 dpi mature SmgshB nodule, there is no more a significant difference compared to the control suggesting that a senescence process begins in control nodules in our conditions. In contrast, $V P E$ expression is significantly induced in SmgshB nodule indicating the progression of the senescence at $20 \mathrm{dpi}$ as observed in the live/dead analysis.

Taken together, our data show that, in contrast to SmGrx1 bacterial mutant (strain 2011), SmgshB mutant (strain 2011) is able to differentiate in bacteroids inside $M$. truncatula cells. The phenotype observed is similar to the one obtained during the interaction of SmgshB mutant (strain 1021) with $M$. sativa (Harrison et al., 2005). In this context, SmGrx1 should use another reducing power than glutathione to perform its activity. We hypothesize that the Grxl could use the $\gamma$-EC accumulated in high quantity in the SmgshB mutant strain as cofactor for the reductive power instead of GSH. Different phenotypes were observed for Arabidopsis thaliana $\gamma$-ECS (gsh1) and GSHS (gsh2) plant mutants. The ghs1 mutant presents an embryo lethal phenotype whereas the gsh2 mutant has a seedling lethal phenotype suggesting a partial replacement of GSH functions by $\gamma$-EC (Cairns et al., 2006; Pasternak et al., 2008). A similar observation is also true for S. meliloti strain 1021 in which the SmgshA mutant strain cannot divide while the SmgshB mutant strain shows a growth delay under free living conditions (Harrison et al., 2005). $\gamma$-EC is also used as a cofactor by glutathione peroxidase- 1 to detoxify ROS in mice (QuintanaCabrera et al., 2012).

The correlation between the GSH content and the BNF in various legumes was the object of several works (Dalton et al., 1986; Evans et al., 1999; Matamoros et al., 1999; Moran et al., 2000; Groten et al., 2005). The plant GSH content is crucial for the bacterial infection effectiveness and the nitrogen fixation efficiency. GSH produced in the plant does not compensate the $g s h B$ mutation suggesting that it is not able to be transported to the bacteroid (Becana et al., 2018). In this context, it should be mentioned that sulfate transport from the plant cell to the bacteroids is very active (Schneider et al., 2019) and that mutation in the sulfate transporter SST1 in Lotus japonicus 
impairs symbiotic nitrogen fixation (Krusell et al., 2005). These data suggest that the sulfur metabolism in bacteroid is depending on sulfate transport and that other sulfur containing molecules such as methionine, cysteine or GSH are not transported through the symbiosome membrane. In this sense, the $R$. etli met $Z$ gene involved in methionine synthesis is required for the efficient interaction with $P$. vulgaris suggesting that methionine is produced by $R$. etli bacteroids during symbiosis, and not supplied by the host cells (Taté et al., 1999). In contrast, the cys $G$ mutant strain of $R$. etli, unable to grow on sulfate as the sole sulfur source, was able to induce nitrogen-fixing nodules on $P$. vulgaris suggesting that cysteine or glutathione is supplied by the plant to bacteria (Taté et al., 1997). Moreover, the nodules induced by cysteine auxotrophs of S. meliloti Rmd201 were fully effective like those of the parental strain-induced nodules suggesting that the alfalfa host provides cysteine to the bacteroid (Abbas et al., 2002). However, even if gshB mutant strains show an impaired symbiotic phenotype, it cannot be excluded that a small fraction of GSH produced in the plant may pass through the bacteroid membrane, partially compensating the bacterial deficiency. The bacterial GSH pool plays also a crucial role for nitrogen fixation efficiency (Figure 5). In the gshB mutant of $S$. meliloti strain 1021, the GSH deficiency was associated with a higher catalase activity in the bacterial mutant in free-living conditions and a reduction of growth efficiency (Harrison et al., 2005). A decrease in the BNF was observed during the symbiosis with $M$. sativa correlated to an early bacteroid death. In $R$. tropici $g s h B$ mutant strain, the lower symbiotic efficiency was associated to an early senescence pattern connected to increased levels of superoxide accumulation (Muglia et al., 2008). For Rhizobium etli gshB mutant, the decrease in BNF was correlated to a strong reduction in nodule number and a deficiency of glutamine transport through Aap and Bra transporter (Taté et al., 2012). This deficiency in nutrient transport also detected in $R$. leguminosarum 3841, which fails to efficiently use various carbon sources such as glucose, succinate, glutamine and histidine, may trigger an early nodule senescence. This transport deficiency in $R$. leguminosarum 3841 gshB mutant strain is also associated to a lower BNF (Cheng et al., 2017). The LysR-type transcriptional regulator LsrB, required for nodulation, also regulates $g s h A$ gene expression through modification of cysteine residues (Luo et al., 2005; Tang et al., 2018) and $g s h B$ gene expression (Lu et al., 2013).

In conclusion, the inoculation of $M$. truncatula with the SmgshB bacterial mutant triggers the development of nodules with a reduced capacity to fix nitrogen. This lower BNF does not seem correlated to a reduced bacteroid differentiation efficiency. However, it is strongly

\section{REFERENCES}

Abbas, B. A., Vineetha, K. E., Prasad, C. K., Vij, N., Hassani, R., and Randhawa, G. S. (2002). Symbiotic characteristics of cysteine and methionine auxotrophs of Sinorhizobium meliloti. Indian J. Exp. Biol. 40, 1121-1130.

Alkhalfioui, F., Renard, M., Frendo, P., Keichinger, C., Meyer, Y., Gelhaye, E., et al. (2008). A novel type of thioredoxin dedicated to symbiosis in legumes. Plant Physiol. 148, 424-435. doi: 10.1104/pp.108.123778 correlated to an early senescence process and the alteration of the expression of multiple nodule gene markers showing the fundamental role of the bacterial GSH pool in nodule functioning.

\section{DATA AVAILABILITY STATEMENT}

The datasets generated for this study are available on request to the corresponding author.

\section{AUTHOR CONTRIBUTIONS}

Conceived and designed the experiments: LY, SE, EB, PF. Performed the experiments: LY, SE, SB, AL, JH, JC, OP, EB. Analyzed the data: LY, SE, EB, PF. Wrote the paper: LY, SE, EB, PF. Amended the paper: SAS, DH.

\section{FUNDING}

LY is supported by a doctoral fellowship from the China scholarship council (CSC). SE was financed by IMAGEEN program from the European Communities and the Tunisian government (bourse d'alternance). This work was supported by the "Institut National de la Recherche Agronomique", the "Centre National de la Recherche Scientifique", the University of Nice-Sophia Antipolis and the French Government (National Research Agency, ANR) through the "STAYPINK" project (reference \# ANR-15-CE20-0005) and the "Investments for the Future" LABEX SIGNALIFE: program reference \# ANR-11LABX-0028-01.

\section{ACKNOWLEDGMENTS}

We are largely indebted to the symbiosis team members for numerous nodule harvests. We thank Renaud Brouquisse for critical reading of the manuscript.

\section{SUPPLEMENTARY MATERIAL}

The Supplementary Material for this article can be found online at: https://www.frontiersin.org/articles/10.3389/fpls.2020.00137/ full\#supplementary-material

Andrio, E., Marino, D., Marmeys, A., De Segonzac, M. D., Damiani, I., Genre, A., et al. (2013). Hydrogen peroxide-regulated genes in the Medicago truncatulaSinorhizobium meliloti symbiosis. New Phytol. 198, 179-189. doi: 10.1111/ nph. 12120

Arthikala, M. K., Sanchez-Lopez, R., Nava, N., Santana, O., Cardenas, L., and Quinto, C. (2014). RbohB, a Phaseolus vulgaris NADPH oxidase gene, enhances symbiosome number, bacteroid size, and nitrogen fixation in nodules and impairs mycorrhizal colonization. New Phytol. 202, 886-900. doi: 10.1111/nph.12714 
Becana, M., Wienkoop, S., and Matamoros, M. (2018). Sulfur transport and metabolism in legume root nodules. Front. Plant Sci. 9, 1434. doi: 10.3389/ fpls.2018.01434

Benyamina, S. M., Baldacci-Cresp, F., Couturier, J., Chibani, K., Hopkins, J., Bekki, A., et al. (2013). Two Sinorhizobium meliloti glutaredoxins regulate iron metabolism and symbiotic bacteroid differentiation. Environ. Microbiol. 15, 795-810. doi: 10.1111/j.1462-2920.2012.02835.x

Berger, A., Boscari, A., Frendo, P., and Brouquisse, R. (2019). Nitric oxide signaling, metabolism and toxicity in nitrogen-fixing symbiosis. J. Exp. Bot. 70, 4505-4520. doi: 10.1093/jxb/erz159

Boisson-Dernier, A., Chabaud, M., Garcia, F., Becard, G., Rosenberg, C., and Barker, D. G. (2001). Agrobacterium rhizogenes-transformed roots of Medicago truncatula for the study of nitrogen-fixing and endomycorrhizal symbiotic associations. Mol. Plant Microbe Interact. 14, 695-700. doi: 10.1094/MPMI.2001.14.6.695

Cárdenas, L., Martínez, A., Sánchez, F., and Quinto, C. (2008). Fast, transient and specific intracellular ROS changes in living root hair cells responding to Nod factors (NFs). Plant J. 56, 802-813. doi: 10.1111/j.1365-313X.2008.03644.x

Cairns, N. G., Pasternak, M., Wachter, A., Cobbett, C. S., and Meyer, A. J. (2006). Maturation of Arabidopsis seeds is dependent on glutathione biosynthesis within the embryo. Plant Physiol. 141, 446-455. doi: 10.1104/pp.106.077982

Cheng, G., Karunakaran, R., East, A. K., Munoz-Azcarate, O., and Poole, P. S. (2017). Glutathione affects the transport activity of Rhizobium leguminosarum 3841 and is essential for efficient nodulation. FEMS Microbiol. Lett. 364, fnx045. doi: 10.1093/femsle/fnx045

Dalton, D. A., Russell, S. A., Hanus, F., Pascoe, G. A., and Evans, H. J. (1986). Enzymatic reactions of ascorbate and glutathione that prevent peroxide damage in soybean root nodules. Proc. Natl. Acad. Sci. U.S.A. 83, 38113815. doi: 10.1073/pnas.83.11.3811

El Msehli, S., Lambert, A., Baldacci-Cresp, F., Hopkins, J., Boncompagni, E., Smiti, S. A., et al. (2011). Crucial role of (homo)glutathione in nitrogen fixation in Medicago truncatula nodules. New Phytol. 192, 496-506. doi: 10.1111/j.14698137.2011.03810.x

Evans, P. J., Gallesi, D., Mathieu, C., Hernandez, M. J., De Felipe, M., Halliwell, B., et al. (1999). Oxidative stress occurs during soybean nodule senescence. Planta 208, 73-79. doi: 10.1007/s004250050536

Foyer, C. H., and Noctor, G. (2011). Ascorbate and glutathione: the heart of the redox hub. Plant Physiol. 155, 2-18. doi: 10.1104/pp.110.167569

Frendo, P., Harrison, J., Norman, C., Hernandez Jimenez, M. J., Van De Sype, G., Gilabert, A., et al. (2005). Glutathione and homoglutathione play a critical role in the nodulation process of Medicago truncatula. Mol. Plant Microbe Interact. 18, 254-259. doi: 10.1094/MPMI-18-0254

Frendo, P., Baldacci-Cresp, F., Benyamina, S. M., and Puppo, A. (2013a). Glutathione and plant response to the biotic environment. Free. Radical. Bio. Med. 65, 724-730. doi: 10.1016/j.freeradbiomed.2013.07.035

Frendo, P., Matamoros, M. A., Alloing, G., and Becana, M. (2013b). Thiol-based redox signaling in the nitrogen-fixing symbiosis. Front. Plant Sci. 4, 376. doi: $10.3389 /$ fpls.2013.00376

Groten, K., Vanacker, H., Dutilleul, C., Bastian, F., Bernard, S., Carzaniga, R., et al. (2005). The roles of redox processes in pea nodule development and senescence. Plant Cell Environ. 28, 1293-1304. doi: 10.1111/j.13653040.2005.01376.x

Haag, A. F., Baloban, M., Sani, M., Kerscher, B., Pierre, O., Farkas, A., et al. (2011). Protection of Sinorhizobium against host cysteine-rich antimicrobial peptides is critical for symbiosis. PloS Biol. 9, e1001169. doi: 10.1371/ journal.pbio. 1001169

Harrison, J., Jamet, A., Muglia, C. I., Van De Sype, G., Aguilar, O. M., Puppo, A., et al. (2005). Glutathione plays a fundamental role in growth and symbiotic capacity of Sinorhizobium meliloti. J. Bacteriol. 187, 168-174. doi: 10.1128/ JB.187.1.168-174.2005

Hichri, I., Boscari, A., Castella, C., Rovere, M., Puppo, A., and Brouquisse, R. (2015). Nitric oxide: a multifaceted regulator of the nitrogen-fixing symbiosis. J. Exp. Bot. 66, 2877-2887. doi: 10.1093/jxb/erv051

Innocenti, G., Pucciariello, C., Le Gleuher, M., Hopkins, J., De Stefano, M., Delledonne, M., et al. (2007). Glutathione synthesis is regulated by nitric oxide in Medicago truncatula roots. Planta 225, 1597-1602. doi: 10.1007/s00425-006-0461-3

Jamet, A., Mandon, K., Puppo, A., and Hérouart, D. (2007). H2O2 is required for optimal establishment of the Medicago sativa/Sinorhizobium meliloti symbiosis. J. Bacteriol. 189, 8741-8745. doi: 10.1128/JB.01130-07
Krusell, L., Krause, K., Ott, T., Desbrosses, G., Krämer, U., Sato, S., et al. (2005). The sulfate transporter SST1 is crucial for symbiotic nitrogen fixation in Lotus japonicus root nodules. Plant Cell. 17, 1625-1636. doi: 10.1105/tpc.104.030106

Livak, K. J., and Schmittgen, T. D. (2001). Analysis of relative gene expression data using real-time quantitative PCR and the 2- $\Delta \Delta \mathrm{CT}$ method. Methods 25, 402408. doi: $10.1006 /$ meth.2001.1262

Lu, D., Tang, G., and Luo, L. (2013). The Sinorhizobium meliloti LysR family transcriptional factor LsrB is involved in the regulation of glutathione biosynthesis. Acta Biochim. Biophys. Sin. (Shanghai) 45, 882-888. doi: 10.1093/abbs/gmt083

Luo, L., Yao, S. Y., Becker, A., Rüberg, S., Yu, G. Q., Zhu, J. B., et al. (2005). Two new Sinorhizobium meliloti LysR-type transcriptional regulators required for nodulation. J. Bacteriol. 187, 4562-4572. doi: 10.1128/JB.187.13.45624572.2005

Marino, D., Andrio, E., Danchin, E. G., Oger, E., Gucciardo, S., Lambert, A., et al. (2011). A Medicago truncatula NADPH oxidase is involved in symbiotic nodule functioning. New Phytol. 189, 580-592. doi: 10.1111/j.14698137.2010.03509.x

Marino, D., Dunand, C., Puppo, A., and Pauly, N. (2012). A burst of plant NADPH oxidases. Trends Plant Sci. 17, 9-15. doi: 10.1016/ j.tplants.2011.10.001

Matamoros, M. A., Moran, J. F., Iturbe-Ormaetxe, I., Rubio, M. C., and Becana, M. (1999). Glutathione and homoglutathione synthesis in legume root nodules. Plant Physiol. 121, 879-888. doi: 10.1104/pp.121.3.879

Mergaert, P., Nikovics, K., Kelemen, Z., Maunoury, N., Vaubert, D., Kondorosi, A., et al. (2003). A novel family in Medicago truncatula consisting of more than 300 nodule-specific genes coding for small, secreted polypeptides with conserved cysteine motifs. Plant Physiol. 132, 161-173. doi: 10.1104/ pp.102.018192

Mergaert, P., Uchiumi, T., Alunni, B., Evanno, G., Cheron, A., Catrice, O., et al. (2006). Eukaryotic control on bacterial cell cycle and differentiation in the Rhizobium-legume symbiosis. Proc. Natl. Acad. Sci. U.S.A. 103, 5230-5235. doi: 10.1073/pnas.0600912103

Moran, J. F., Iturbe-Ormaetxe, I., Matamoros, M. A., Rubio, M. C., Clemente, M. R., Brewin, N. J., et al. (2000). Glutathione and homoglutathione synthetases of legume nodules. cloning, expression, and subcellular localization. Plant Physiol. 124, 13811392. doi: $10.1104 /$ pp.124.3.1381

Muglia, C., Comai, G., Spegazzini, E., Riccillo, P. M., and Aguilar, O. M. (2008). Glutathione produced by Rhizobium tropici is important to prevent early senescence in common bean nodules. FEMS Microbiol. Lett. 286, 191-198. doi 10.1111/j.1574-6968.2008.01285.x

Ott, T., Van Dongen, J. T., Gu, C., Krusell, L., Desbrosses, G., Vigeolas, H., et al. (2005). Symbiotic leghemoglobins are crucial for nitrogen fixation in legume root nodules but not for general plant growth and development. Curr. Biol. 15, 531-535. doi: 10.1016/j.cub.2005.01.042

Pasternak, M., Lim, B., Wirtz, M., Hell, R., Cobbett, C. S., and Meyer, A. J. (2008). Restricting glutathione biosynthesis to the cytosol is sufficient for normal plant development. Plant J. 53, 999-1012. doi: 10.1111/j.1365313X.2007.03389.x

Pierre, O., Hopkins, J., Combier, M., Baldacci, F., Engler, G., Brouquisse, R., et al. (2014). Involvement of papain and legumain proteinase in the senescence process of Medicago truncatula nodules. New Phytol. 202, 849-863. doi: $10.1111 /$ nph.12717

Puppo, A., Pauly, N., Boscari, A., Mandon, K., and Brouquisse, R. (2013). Hydrogen peroxide and nitric oxide: key regulators of the LegumeRhizobium and mycorrhizal symbioses. Antioxid. Redox Sign. 18, 2202-2219. doi: 10.1089 /ars.2012.5136

Quintana-Cabrera, R., Fernandez-Fernandez, S., Bobo-Jimenez, V., Escobar, J., Sastre, J., Almeida, A., et al. (2012). $\gamma$-Glutamylcysteine detoxifies reactive oxygen species by acting as glutathione peroxidase-1 cofactor. Nat. Commun. 3, 718. doi: $10.1038 /$ ncomms 1722

Ribeiro, C. W., Alloing, G., Mandon, K., and Frendo, P. (2015). Redox regulation of differentiation in symbiotic nitrogen fixation. Biochim. Biophys. Acta 1850, 1469-1478. doi: 10.1016/j.bbagen.2014.11.018

Ribeiro, C. W., Baldacci-Cresp, F., Pierre, O., Larousse, M., Benyamina, S., Lambert, A., et al. (2017). Regulation of differentiation of nitrogen-fixing bacteria by microsymbiont targeting of plant thioredoxin s1. Curr. Biol. 27, 250-256. doi: 10.1016/j.cub.2016.11.013 
Rouhier, N., Lemaire, S. D., and Jacquot, J.-P. (2008). The role of glutathione in photosynthetic organisms: emerging functions for glutaredoxins and glutathionylation. Annu. Rev. Plant Biol. 59, 143-166. doi: 10.1146/ annurev.arplant.59.032607.092811

Santos, R., Hérouart, D., Sigaud, S., Touati, D., and Puppo, A. (2001). Oxidative burst in alfalfa-Sinorhizobium meliloti symbiotic interaction. Mol. Plant Microbe Interact. 14, 86-89. doi: 10.1094/MPMI.2001.14.1.86

Schindelin, J., Arganda-Carreras, I., Frise, E., Kaynig, V., Longair, M., Pietzsch, T., et al. (2012). Fiji: an open-source platform for biological-image analysis. Nat. Methods 9, 676-682. doi: 10.1038/nmeth.2019

Schneider, S., Schintlmeister, A., Becana, M., Wagner, M., Woebken, ,. D., and Wienkoop, S. (2019). Sulfate is transported at significant rates through the symbiosome membrane and is crucial for nitrogenase biosynthesis. Plant Cell Environ. 42, 1180-1189. doi: 10.3389/fpls.2018.01434

Tang, G., Li, N., Liu, Y., Yu, L., Yan, J., and Luo, L. (2018). Sinorhizobium meliloti glutathione reductase is required for both redox homeostasis and symbiosis. Appl. Environ. Microb. 84, e01937-e01917. doi: 10.1128/aem.01937-17

Taté, R., Riccio, A., Iaccarino, M., and Patriarca, E. J. (1997). A cysG mutant strain of Rhizobium etli pleiotropically defective in sulfate and nitrate assimilation. J. Bacteriol. 179, 7343-7350. doi: 10.1128/jb.179.23.7343-7350.1997

Taté, R., Riccio, A., Caputo, E., Iaccarino, M., and Patriarca, E. J. (1999). The Rhizobium etli metZ gene is essential for methionine biosynthesis and nodulation of Phaseolus vulgaris. Mol. Plant Microbe Interact. 1999 12, 2434. doi: 10.1094/MPMI.1999.12.1.24
Taté, R., Cermola, M., Riccio, A., Diez-Roux, G., and Patriarca, E. J. (2012) Glutathione is required by Rhizobium etli for glutamine utilization and symbiotic effectiveness. Mol. Plant Microbe Interact. 25, 331-340. doi: 10.1094/MPMI-06-11-0163

Trinchant, J.-C., Boscari, A., Spennato, G., Van de Sype, G., and Le Rudelier, D. (2004). Proline betaine accumulation in alfalfa plants under sodium chloride stress. Exploring its compartmentalization in nodules. Plant Physiol. 135, 1583-1594. doi: 10.1104/pp.103.037556

Xi, J., Chen, Y., Nakashima, J., Wang, S. M., and Chen, R. (2013). Medicago truncatula esn 1 defines a genetic locus involved in nodule senescence and symbiotic nitrogen fixation. Mol. Plant Microbe Interact. 26, 893-902. doi: 10.1094/MPMI-02-13-0043-R

Conflict of Interest: The authors declare that the research was conducted in the absence of any commercial or financial relationships that could be construed as a potential conflict of interest.

Copyright (C) 2020 Yang, El Msehli, Benyamina, Lambert, Hopkins, Cazareth, Pierre, Hérouart, Achi-Smiti, Boncompagni and Frendo. This is an open-access article distributed under the terms of the Creative Commons Attribution License (CC BY). The use, distribution or reproduction in other forums is permitted, provided the original author(s) and the copyright owner(s) are credited and that the original publication in this journal is cited, in accordance with accepted academic practice. No use, distribution or reproduction is permitted which does not comply with these terms. 